\title{
Measuring the reactivity of a silicon-terminated probe
}

\author{
Adam Sweetman, ${ }^{1, *}$ Julian Stirling, ${ }^{2}$ Samuel Paul Jarvis, ${ }^{1}$ Philipp Rahe,,${ }^{1}$ and Philip Moriarty ${ }^{1}$ \\ ${ }^{1}$ The School of Physics and Astronomy, The University of Nottingham, Nottingham NG7 2RD, England, United Kingdom \\ ${ }^{2}$ NIST, Mass and Force Group, 100 Bureau Drive, Gaithersburg, Maryland 20899, USA
}

(Received 4 April 2016; revised manuscript received 18 August 2016; published 30 September 2016)

\begin{abstract}
It is generally accepted that the exposed surfaces of silicon crystals are highly reactive due to the dangling bonds which protrude into the vacuum. However, surface reconstruction not only modifies the reactivity of bulk silicon crystals, but also plays a key role in determining the properties of silicon nanocrystals. In this study we probe the reactivity of silicon clusters at the end of a scanning probe tip by examining their interaction with closed-shell fullerene molecules. Counter to intuitive expectations, many silicon clusters do not react strongly with the fullerene cage, and we find that only specific highly oriented clusters have sufficient reactivity to break open the existing carbon-carbon bonds.
\end{abstract}

DOI: 10.1103/PhysRevB.94.115440

\section{INTRODUCTION}

Recent developments in scanning probe microscopy now allow for the almost-routine intramolecular imaging of planar organic molecules using functionalized tips on metallic surfaces at liquid helium temperatures [1]. Very recently it has been shown that similar contrast can also be obtained on semiconductor surfaces at liquid nitrogen temperatures [2,3] and even at room temperature [4]. In experiments conducted on metallic surfaces it is widely assumed that intramolecular resolution can only be obtained via functionalization of the scanning probe tip with an unreactive atom or molecule, since otherwise the high reactivity of the metallic tip causes manipulation of the molecule before the tip is able to enter into the Pauli repulsion regime where intramolecular contrast is obtained. However, in recent combined experimental and theoretical studies, it has been suggested that semiconductor tips may counterintuitively be able to provide similar resolution [2-4], despite silicon traditionally being thought of as a highly reactive tip material [5-7].

In this study we examine the reactivity of a number of silicon- and metal-terminated probes with the buckminster fullerene $\left(\mathrm{C}_{60}\right)$ cage by measuring the peak attractive force between the probe and $\mathrm{C}_{60}$ molecule during site-specific force spectroscopy [8] experiments. Surprisingly, we find that many, otherwise reactive, silicon-terminated clusters have an interaction with the carbon cage that is comparable to the interaction measured with $\mathrm{CO}$ - or fullerene-terminated probes [9-11] and are similarly able to probe the repulsive part of the tip-sample interaction without changes in the tip or manipulation of the molecule. We address the experimental challenges in determining the tip termination by inverse imaging of the tip on well-defined features, and the use of combined scanning tunneling microscopy and noncontact atomic force microscopy (STM/NC-AFM) imaging. We interpret our experimental

\footnotetext{
*adam.sweetman@nottingham.ac.uk
}

Published by the American Physical Society under the terms of the Creative Commons Attribution 3.0 License. Further distribution of this work must maintain attribution to the author(s) and the published article's title, journal citation, and DOI. results with support from $a b$ initio density functional theory (DFT) calculations and compare our data to simulations investigating the interaction of metal and silicon clusters with carbon nanotubes $[12,13]$ and recent intramolecular imaging results on semiconductor substrates $[2,4]$.

\section{EXPERIMENTAL METHODS}

Clean $\mathrm{Si}(111)-7 \times 7$ surfaces were prepared by flash annealing a silicon wafer to $1200^{\circ} \mathrm{C}$, rapid cooling to $900^{\circ} \mathrm{C}$, and then slow cooling to room temperature, ensuring pressures below $10^{-10}$ mbar throughout. Clean $\mathrm{Ag}(111)-1 \times 1$ surfaces were prepared by standard argon sputter-anneal cycles with a beam energy of $1 \mathrm{keV}$ and an anneal temperature of $500^{\circ} \mathrm{C}$. Low coverages of $\mathrm{C}_{60}$ were prepared by depositing the molecules from a homemade tantalum pocket deposition source onto the substrate. During deposition the $\mathrm{Si}(111)-7 \times 7$ was at room temperature, whereas the $\operatorname{Ag}(111)$ was cooled to $\sim 100 \mathrm{~K}$. On $\operatorname{Ag}(111)$ the molecules formed small, closepacked islands, whereas on $\mathrm{Si}(111)-7 \times 7$ isolated molecules were found due to the strong molecule-substrate interaction [14]. Postdeposition, the sample was transferred into the scan head of an Omicron Nanotechnology [15] LT NC-AFM operating in UHV at cryogenic temperatures, and left to cool to 77 or $5 \mathrm{~K}$ before imaging.

Commercial qPlus sensors (Omicron $\mathrm{GmbH}$ ) with electrochemically etched tungsten wire tips were introduced into the scan head without any further preparation. The sensors were first prepared on clean $\mathrm{Si}(111)-7 \times 7$ surfaces by standard STM techniques until good STM/NC-AFM resolution was achieved. Detailed information on the termination of the tip apex is given for each dataset. Single $\Delta f(z)$ measurements were performed both on and off the molecules and site-specific (short-range) $F(z)$ curves were extracted using the "on-off" method $[8,16]$ and the Sader-Jarvis algorithm [17].

\section{SIMULATION METHODS}

Simulated spectroscopy calculations were performed using the open source CP2K/Quickstep DFT code $[18,19]$ utilizing a hybrid Gaussian and plane-wave method [20]. The Goedecker, Teter, and Hutter pseudopotentials [21] and Perdew-Burke- 
Ernzerhof generalized gradient approximation method [22] were used with a 300-Ry plane-wave energy cutoff. Dispersion interactions were taken into account by employing the Grimme DFT-D3 method [23]. A double-zeta Gaussian basis set plus polarization (DZVP-MOLOPT) [24] was used using a force convergence criterion for geometry relaxation of $0.05 \mathrm{eV} / \AA$.

\section{EXPERIMENTAL RESULTS}

A key aspect of this study is ensuring a high degree of confidence in the termination of the scanning probe tip. It is well known that molecules adsorbed on both metallic and semiconducting surfaces are easily transferred to the tip during normal STM/AFM imaging. Therefore, in cases where the tip termination is crucial for data interpretation, it is inadequate to rely on crashing the tip into the regions of the pristine surface, as surface adsorbed molecules may still be inadvertently transferred to the tip during this procedure. Consequently, some independent means of checking the termination must be used.

In the first dataset [25] (Fig. 1) a clean $\mathrm{Si}(111)-7 \times 7$ surface was prepared as described in the methods section. A prepare-retract-deposit-approach-check approach [26] was then used to identify a silicon-terminated apex. In detail, the surface was first approached with a fresh qPlus sensor and the tip indented heavily into the surface with multiple voltage pulses to ensure the tip was coated in silicon. Importantly, there were no $\mathrm{C}_{60}$ molecules on the surface at this time to potentially contaminate the tip apex. We then characterized the NC-AFM imaging and force interaction on the silicon adatoms (i.e., using the $\mathrm{Si}(111)-7 \times 7$ adatoms as a "calibration" sample for the tip [10]), and note that the imaging and force spectroscopy values observed are consistent with literature values $[8,27]$ for silicon-terminated tips on the $\operatorname{Si}(111)-7 \times 7$ adatoms. This also confirms that the tip was not terminated with, for example, common vacuum contaminants such as $\mathrm{CO}$ or $\mathrm{OH}$.

The tip was withdrawn and the sample transferred to a separate preparation chamber, where a low coverage of $\mathrm{C}_{60}$ was prepared as described above. The sample was then reintroduced into the scan head, and the tip carefully reapproached at low $\Delta f$ setpoint. Once approached, the sample was scanned at gradually increasing $\Delta f$ setpoint until molecular resolution was obtained. The tip was then recharacterized on the Si(111)$7 \times 7$ by imaging and force spectroscopy of the adatoms, before performing force spectroscopy on the adsorbed $\mathrm{C}_{60}$ molecules. By comparing the imaging and tip-sample forces on the $\mathrm{Si}(111)-7 \times 7$ adatoms before, and after, the $\mathrm{C}_{60}$ deposition, we can confirm that no identifiable change in the tip apex has occurred, and so therefore have a high degree of confidence that the tip is silicon terminated. We also note the slight noncircular appearance of the adatoms, which is suggestive of a tip with some degree of asymmetry (e.g., a dimerlike termination). Surprisingly, the largest attractive tip-sample force between the silicon tip and $\mathrm{C}_{60}$ molecule is of the order $100-300 \mathrm{pN}$, comparable to measurements performed with $\mathrm{CO}$ or $\mathrm{C}_{60}$ terminated tips $[9,28]$ and far below the peak attractive forces detected during previous force measurements of the $\mathrm{C}_{60}-\mathrm{Si}(111)-7 \times 7$ adatom interaction [10], or between a $\mathrm{C}_{60}$ molecule and $\mathrm{Cu}$ cluster [11].
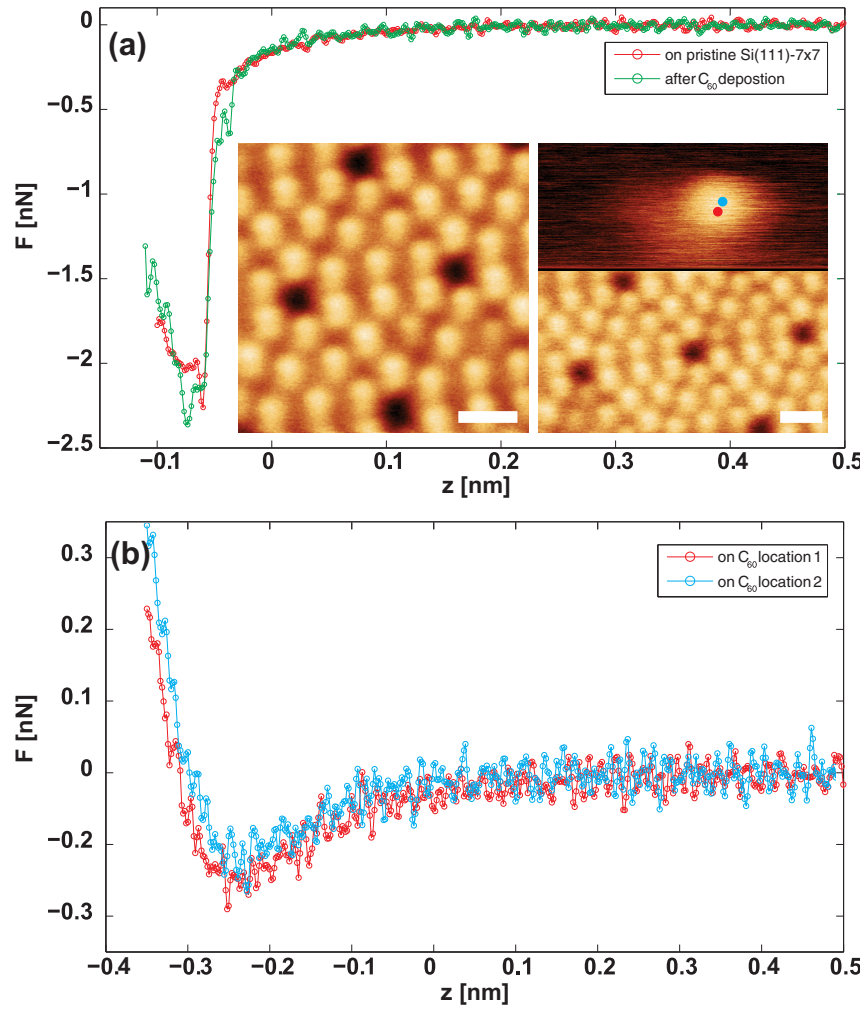

FIG. 1. Imaging and spectroscopy performed with the same tip before and after $\mathrm{C}_{60}$ deposition. (a) Site-specific $\mathrm{F}(z)$ curves taken on a $\mathrm{Si}(111)-7 \times 7$ silicon adatom, before (red) and after (green) retraction of the tip, removal of the sample, $\mathrm{C}_{60}$ deposition, and reapproach. Inset: Left, constant $\Delta f$ image of clean $\mathrm{Si}(111)-7 \times 7$ surface; right, constant $\Delta f$ image of $\mathrm{Si}(111)-7 \times 7$ and $\mathrm{C}_{60}$ molecule after deposition. Note that both the quantitative tip-sample force and qualitative appearance of the adatoms remains the same, indicating the same tip state has been preserved. (b) Site-specific $\mathrm{F}(z)$ curves taken over two positions on the $\mathrm{C}_{60}$ molecule (locations marked on the image) with the same silicon-terminated tip. Image parameters: $\Delta f=-9 \mathrm{~Hz}$ (over $\mathrm{Si}$ ), $\Delta f=-7.5 \mathrm{~Hz}$ (over $\mathrm{Si}$ ) and $-1.7 \mathrm{~Hz}$ (over $\left.\mathrm{C}_{60}\right), \mathrm{A}_{0}=110 \mathrm{pm}, \mathrm{V}_{\text {gap }}=0 \mathrm{~V}$. Scale bar $1 \mathrm{~nm}$. Data acquired at $77 \mathrm{~K}$.

Although this method gives the highest certainty of a silicon-terminated tip, it is prohibitively time consuming for normal scanning probe operation, as any slight change in the tip requires the entire procedure to be repeated. However, utilizing these results as a reference, we can interpret data arising from tips with similar properties (but prepared on the surface after $\mathrm{C}_{60}$ deposition) as arising from silicon-terminated tips with a greater degree of confidence, provided the tips are characterized on the $\mathrm{Si}(111)-7 \times 7$ adatoms.

In Fig. 2 we show how a combination of the information extracted via STM and NC-AFM can be used to characterize the tip termination with a high degree of confidence, and demonstrate the reproducibility of the force measurements using silicon-terminated tips (prepared by normal tip conditioning on a surface with a low coverage of $\mathrm{C}_{60}$ ). Here we first use the characteristic appearance of the $\mathrm{C}_{60}$ orbitals in STM imaging to determine that the tip has a $p$ - or $s$-wave-like character. We note that passivated tips (e.g., $\mathrm{OH}$ or $\mathrm{CO}$ 

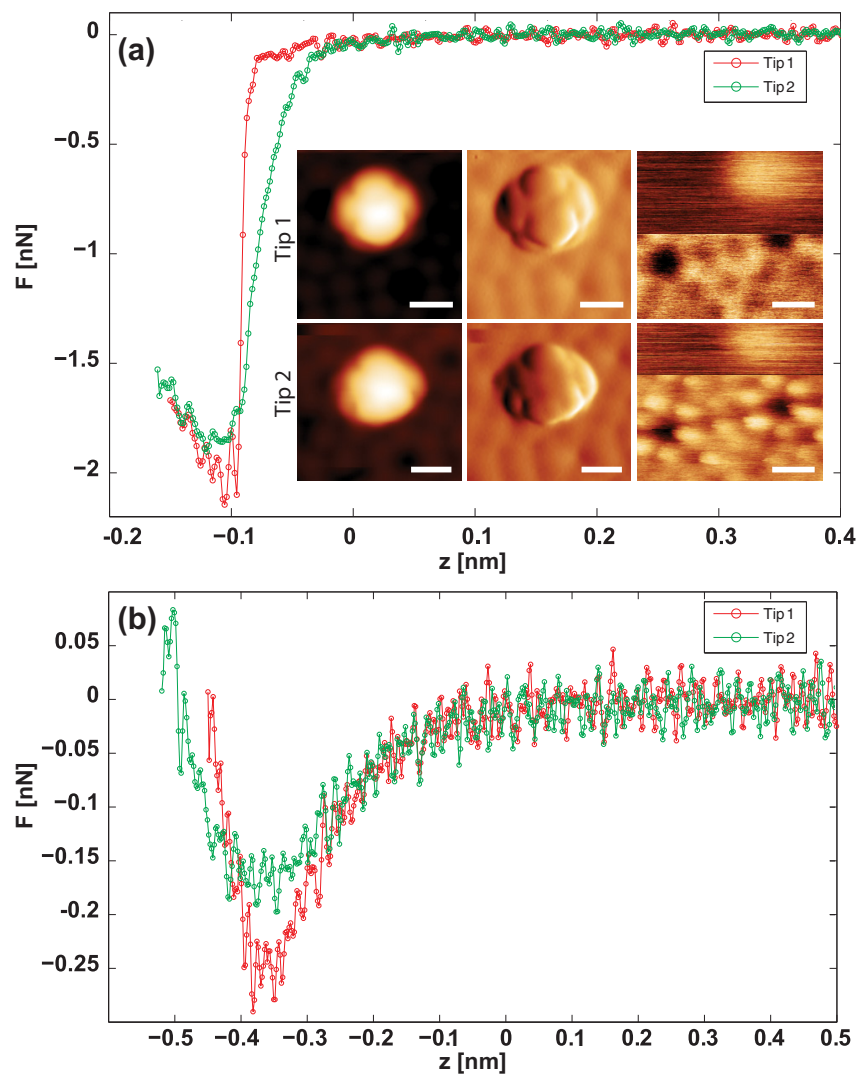

FIG. 2. Data acquired with two probable Si terminated tips. (a) Site-specific $\mathrm{F}(z)$ curves taken over a silicon adatom with Tip 1 (red) and Tip 2 (green). Note the peak forces are larger than we would expect for $\mathrm{C}_{60}$ terminated tip [10]. Inset: top row, L-R dynamic STM (dSTM) topography, $\mathrm{I}_{t}$ (error signal), and constant $\Delta f$ topography of $\mathrm{C}_{60}$ molecule and silicon substrate taken with Tip 1. Bottom row L-R: as for top row but with Tip 2. Tip 1 shows $s$ - or $p$-wave-like dSTM imaging over the $\mathrm{C}_{60}$ molecule and a siliconlike force interaction on the silicon adatoms. After a tip change we observed a slight change in the imaging and force interaction which we term Tip 2. (b) Sitespecific $\mathrm{F}(z)$ curves taken over the same $\mathrm{C}_{60}$ molecule with Tip 1 (red) and Tip 2 (green). Image parameters (dSTM): $\left\langle I_{t}\right\rangle=20 \mathrm{pA}, \mathrm{V}_{\text {gap }}=$ $-2.5 \mathrm{~V}, \mathrm{~A}_{0}=550 \mathrm{pm}$. Image parameters (NC-AFM): $\Delta f=-4$ and $-4.8 \mathrm{~Hz}$ (over $\mathrm{Si}$ ) and $-1 \mathrm{~Hz}\left(\right.$ over $\left.\mathrm{C}_{60}\right), \mathrm{A}_{0}=110 \mathrm{pm}, \mathrm{V}_{\text {gap }}=0 \mathrm{~V}$. Scale bar $1 \mathrm{~nm}$. Data acquired at $77 \mathrm{~K}$.

terminated) could also produce similar STM imaging contrast $[9,29]$, but that a $\mathrm{C}_{60}$-terminated tip would produce a more complex convoluted orbital pattern [30]. The tip apex was also characterized by NC-AFM imaging and force spectroscopy of the Si adatoms [Fig. 2(a)]. The imaging, and large peak attractive forces, show that the tip is not passivated by $\mathrm{CO}$ or $\mathrm{OH}$, since this would result in inverted imaging of the adatoms, and/or peak tip-sample tip-adatom forces of the order of $\sim 100 \mathrm{pN}$ [31-33]. The combination of STM and NC-AFM characterization consequently strongly suggests a silicon-terminated tip apex, particularly in light of the data presented in Fig. 1. This procedure was repeated after we observed a slight change in the contrast [lower row Fig. 2(a)], likely resulting from a slight rearrangement in the atomic structure of the tip apex, and revealed similar characteristics.
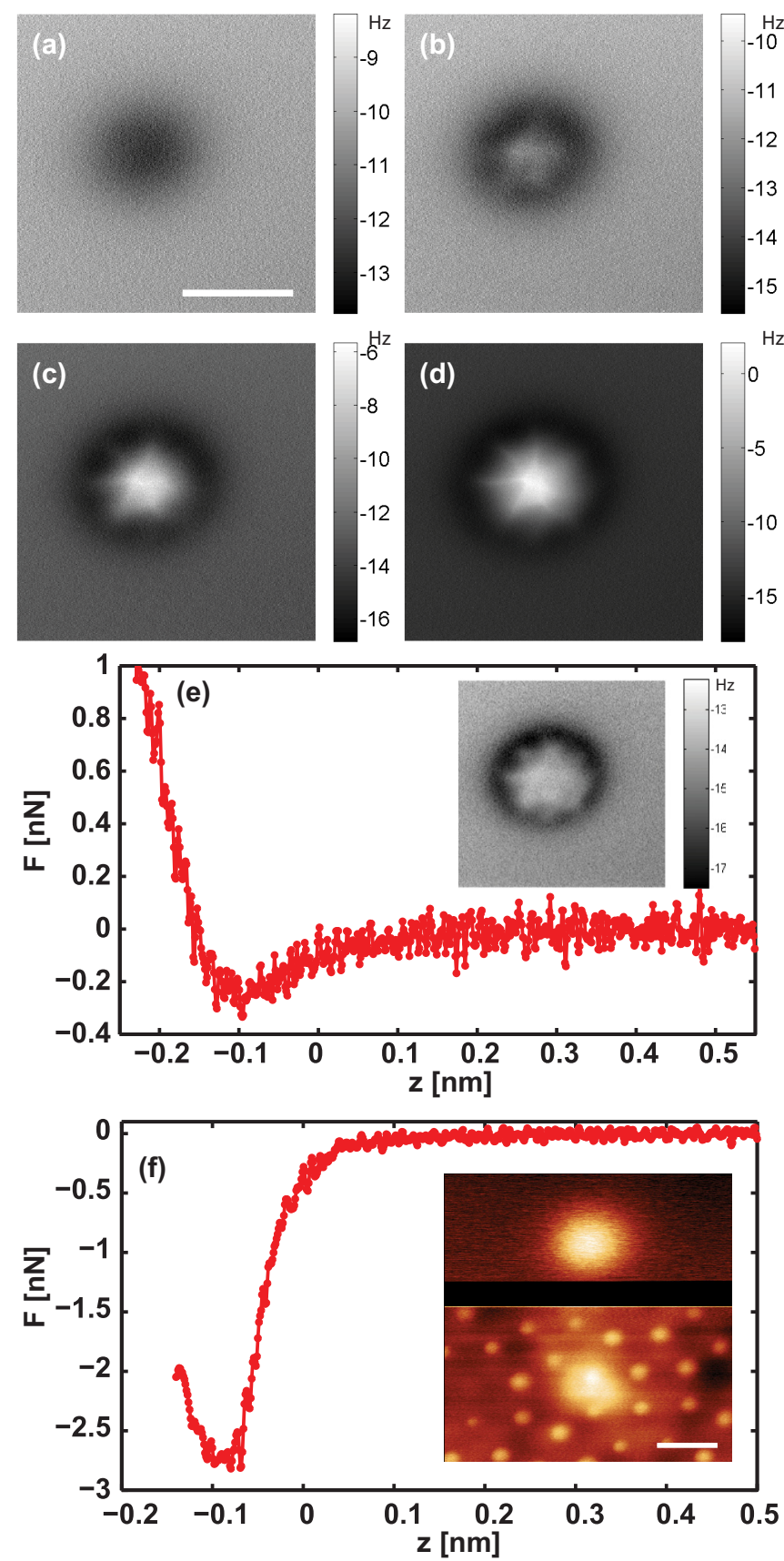

FIG. 3. Constant-height $\Delta f$ images of $\mathrm{C}_{60}$ molecule with a probable Si-terminated tip at (a) $\Delta z=-0.09 \mathrm{~nm}$, (b) $\Delta z=-0.16 \mathrm{~nm}$, (c) $\Delta z=-0.21 \mathrm{~nm}$, (d) $\Delta z=-0.25 \mathrm{~nm}$. (e,f) Site-specific $\mathrm{F}(z)$ curves taken over $\mathrm{C}_{60}$ molecule and $\mathrm{Si}$ adatom (respectively) with the same tip. Inset (e): $\Delta f_{\min }$ image [34] extracted from constant-height image sequence. Inset (f): Constant- $\Delta f$ topography images of silicon substrate and $\mathrm{C}_{60}$ molecule. Image parameters (NC-AFM): $\Delta f$ $=-36.5 \mathrm{~Hz}($ over $\mathrm{Si})$ and $-9.5 \mathrm{~Hz}\left(\right.$ over $\left.\mathrm{C}_{60}\right), \mathrm{A}_{0}=270 \mathrm{pm}$, $\mathrm{V}_{\text {gap }}=0 \mathrm{~V}$. Scale bars $1 \mathrm{~nm}$. Data acquired at $77 \mathrm{~K}$.

Importantly, for both tip states we observed peak tip-molecule forces on the order of $100-250 \mathrm{pN}$, which is consistent with the forces for the silicon-terminated tip characterized in Fig. 1.

In Fig. 3 we highlight the ability of a silicon-terminated tip to probe the tip-molecule interaction at close approach 
by presenting an additional dataset taken with a tip with the same NC-AFM characteristics as those in Figs. 1 and 2 (i.e., strong interaction with silicon adatoms, no evidence of $\mathrm{C}_{60}$ cage features during NC-AFM imaging of the adatoms, and a weak tip-molecule interaction), which we therefore also assign as being silicon terminated. By performing constant-height imaging at a range of tip-sample separations, it is possible to see that the evolution in the constant-height slices, and also the contrast in the $\Delta f_{\text {min }}$ image (showing the variation in the minimum in $\Delta f$ at each lateral position over the molecule [34]), is similar to that observed in intramolecular imaging experiments with molecular terminated tips [1,34], confirming that the tip is able to probe the repulsive branch of the force curve over the entire region of the molecule.

We briefly mention that the contrast in the constant-height slices presented in Fig. 3 does not show clear bondlike resolution at close approach such that the orientation of the molecule can be determined. This must be reconciled with other results showing that silicon-terminated probes can resolve the structure of planar organic molecules [2,4]. Most likely the contrast at close approach is the result of multiatom interactions between the tip apex and the $\mathrm{C}_{60}$ molecule. Similar contrast has been observed during constant-height imaging of $\mathrm{C}_{60}$ molecules with $\mathrm{C}_{60}$ terminated tips [34], where the well-characterized nature of the tip apex means that the contrast can be conclusively assigned as resulting from multiatom interactions between the two molecules. We hypothesize that the tip used in Fig. 3 has a structure such that the back-bonding silicon atoms of the tip are able to interact with the $\mathrm{C}_{60}$ molecule during close approach in a similar fashion, which may explain the qualitatively similar contrast. We also note that the high curvature of the $\mathrm{C}_{60}$ molecule and the large number of possible orientations on the $\mathrm{Si}(111)$ surface present a particularly challenging case for obtaining intramolecular contrast.

In order to interpret our data we performed simulated force spectroscopy, modeling (a) the interaction between a $\mathrm{C}_{60}$ molecule and prototypical silicon clusters and (b) the interaction between different silicon clusters, using the Quickstep/CP2K code. We used a T4 cluster to approximate the $\operatorname{Si}(111)-7 \times 7$ adatom [35], and a dimerized tip cluster to approximate a typical silicon-terminated probe tip [36]. To obtain simulated $F(z)$ curves, the silicon cluster was initially positioned at a vertical distance of $0.8 \mathrm{~nm}$ above a hexagon atom of the $\mathrm{C}_{60}$ molecule (see inset of Fig. 4 for schematic representations of the tip-molecule geometries), and the cluster was then moved towards the molecule in quasistatic steps to just beyond the force turning point. At each step the geometry of the system was optimized until the forces on the nonfixed molecule and cluster atoms were no larger than $0.05 \mathrm{eV} / \mathrm{A}$. The vertical force between cluster and molecule was then calculated by numerical differentiation of the energy.

The results of these simulations are shown in Fig. 4, and serve to highlight the differences in the interaction between different types of silicon cluster and the $\mathrm{C}_{60}$ molecule. The results of the simulations with the $T 4$ cluster and the $\mathrm{C}_{60}$ molecule reproduce the situation where a scanning probe tip is functionalized with a $\mathrm{C}_{60}$ molecule $[10,34]$ and sitespecific force spectroscopy is performed over the adatoms of
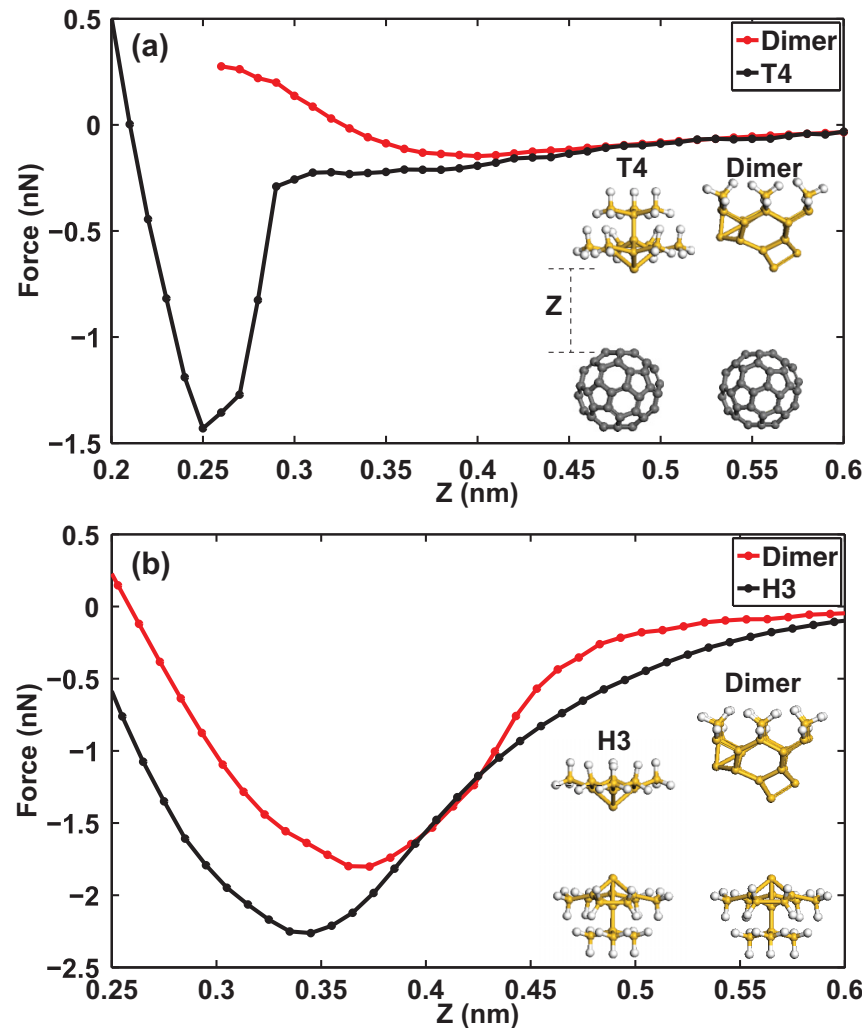

FIG. 4. (a) Simulated force-distance approach curves calculated for a $\mathrm{T} 4$ and dimerized silicon cluster placed over a hexagon atom of a $\mathrm{C}_{60}$ molecule. Inset: Ball and stick models showing the geometries for the $\mathrm{T} 4$ and dimer tip clusters and the $\mathrm{C}_{60}$ molecule. (b) Simulated force-distance approach curves for a reactive $\mathrm{H} 3$ tip and the dimerized silicon tip placed over the $\mathrm{T} 4$ silicon adatom cluster. Inset: Ball and stick models showing the geometries for the silicon clusters.

the $\operatorname{Si}(111)-7 \times 7$ surface. In this situation large tip-sample forces (on the order of $1.5 \mathrm{nN}$ ) are measured experimentally, suggestive of a strong chemical interaction between the $C_{60}$ molecule and silicon atom. This finding is also in line with the known chemisoption of fullerenes on silicon surfaces [14].

However, we find experimentally that for most siliconterminated probe tips we do not observe these strong interactions, and we are able to reproducibly approach close to the molecule and probe the repulsive branch of the tip-sample force curve. This is in line with the evolution observed with the dimer tip and $\mathrm{C}_{60}$ molecule simulations, where we observe peak attractive tip-sample forces on the order of $150 \mathrm{pN}$. While experimentally the exact configuration of the silicon cluster at our tip apex is unknown, this strongly suggests that silicon tips of this "class" exhibit the behavior we observe experimentally.

We also simulate the $F(z)$ characterization of our siliconterminated tips on the $\mathrm{Si}(111)-7 \times 7$ adatoms by modeling the interaction of the dimerized tip cluster, and a reactive H3 cluster, with the T4 cluster representing the $\operatorname{Si}(111)-7 \times 7$ adatom [Fig. 4(b)]. We find that the interaction of the dimerized tip with the T4 adatom cluster is only slightly quantitatively different to the interaction of the more reactive $\mathrm{H} 3$ tip cluster, with both tips interacting strongly with the T4 cluster. This highlights how a simple qualitative characterization on the T4 

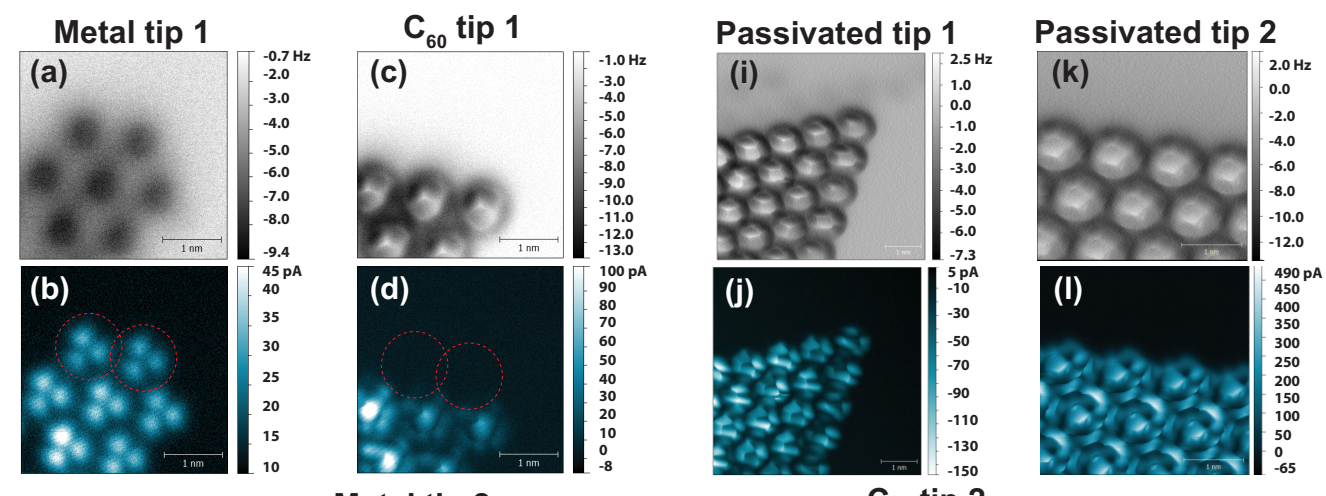

Metal tip 2
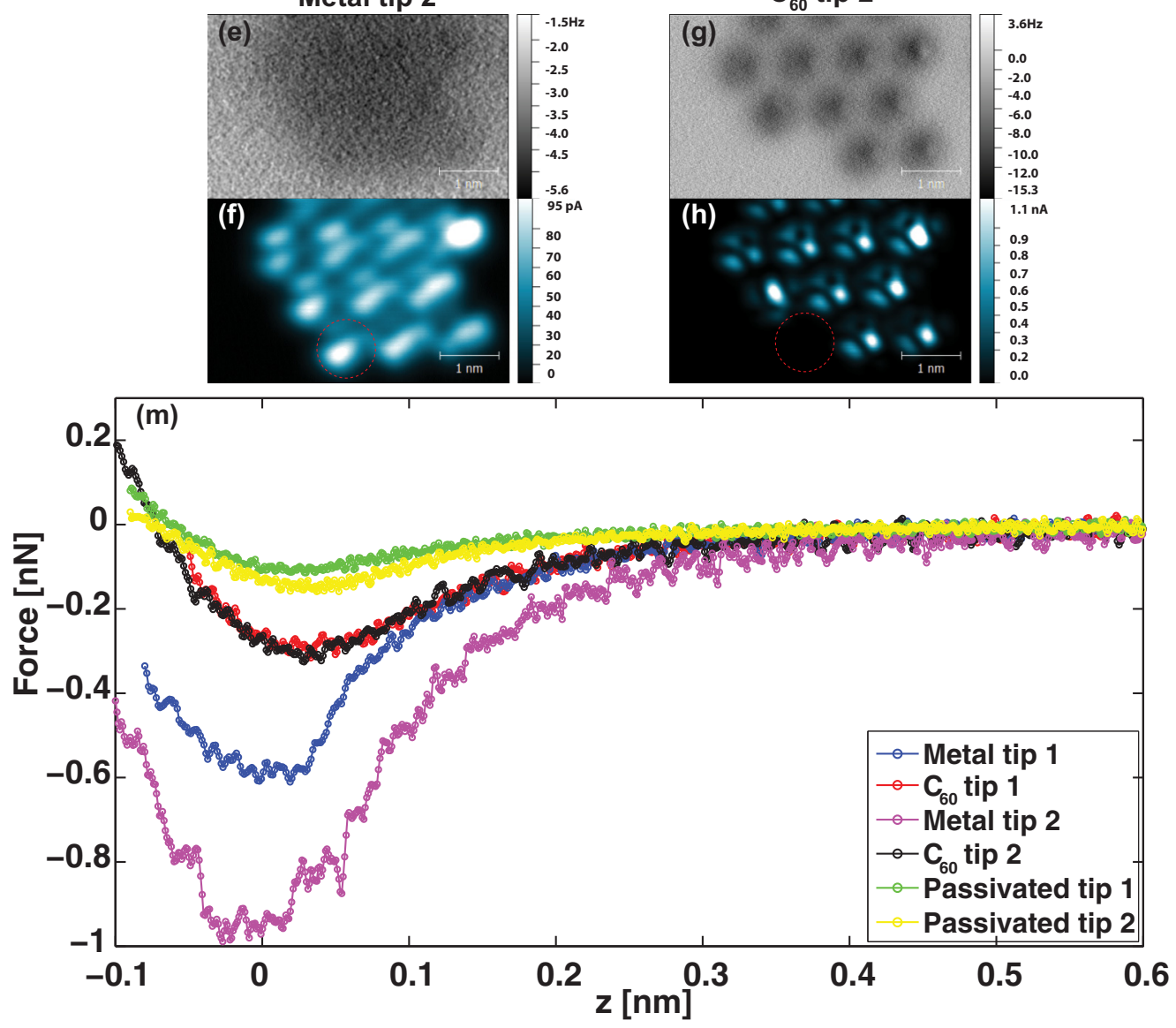

FIG. 5. (a) Simultaneous constant-height $\Delta f$ and (b) tunnel current data acquired over a $\mathrm{C}_{60}$ island with metal terminated tip 1. Image parameters: $\mathrm{A}_{0}=70 \mathrm{pm}, \mathrm{V}_{\text {gap }}=50 \mathrm{mV}$. After close approach two $\mathrm{C}_{60}$ molecules were removed from the island (marked by red circles), and likely transferred to the tip apex. (c) Constant-height $\Delta f$ and (d) tunnel current data acquired over the same area with the probable $\mathrm{C}_{60}$-terminated tip 1. Image parameters: $\mathrm{A}_{0}=70 \mathrm{pm}, \mathrm{V}_{\text {gap }} \sim 0 \mathrm{~V}$. (e) Simultaneous constant-height $\Delta f$ and (f) tunnel current data acquired over a $\mathrm{C}_{60}$ island with metal terminated tip $2 . \mathrm{A}_{0}=110 \mathrm{pm}, \mathrm{V}_{\text {gap }}=100 \mathrm{mV}$. After close approach to the $\mathrm{C}_{60}$ molecule highlighted with a red broken circle, the molecule was adsorbed onto the tip. (g) Constant-height $\Delta f$ and tunnel current (h) data acquired over the same area with $\mathrm{C}_{60}$-terminated tip 2. $\mathrm{A}_{0}=110 \mathrm{pm}, \mathrm{V}_{\text {gap }}=10 \mathrm{mV}$. (i) Constant-height $\Delta f$ and (j) simultaneous tunnel current data acquired over a $\mathrm{C}_{60}$ island with probable $\mathrm{CO}$ (or similar)-terminated tip $1 . \mathrm{A}_{0}=70 \mathrm{pm}, \mathrm{V}_{\text {gap }}=-90 \mu \mathrm{V}$. (k) Constant-height $\Delta f$ and (1) simultaneous tunnel current data acquired over a $\mathrm{C}_{60}$ island with probable $\mathrm{CO}$ (or similar)-terminated tip 2 . $\mathrm{A}_{0}=110 \mathrm{pm}, \mathrm{V}_{\text {gap }}=10 \mathrm{mV}$. (m) Site-specific $\mathrm{F}(z)$ curves acquired over $\mathrm{C}_{60}$ molecules with each tip. The absolute $z$ axis of each curve has been shifted to approximately align the minimums in the force curves. All data acquired at $5 \mathrm{~K}$.

adatom is insufficient to definitively identify which silicon clusters will react with the fullerene cage, as experimentally we observe a wide range of tip-silicon forces (approximately $1.8-2.8 \mathrm{nN}$ ) for tips that do not interact strongly with the $\mathrm{C}_{60}$ molecule.

\section{Measurements on $\operatorname{Ag}(111)$}

Most intramolecular NC-AFM imaging has thus far been performed on metallic substrates, using functionalised metal tips, whereas imaging intramolecular features and direct measurement of intermolecular forces on semiconductor substrates 
is a relatively new field of research [2-4]. Consequently, tip-molecule interactions are less well understood on semiconducting surfaces, as a result of the semiconducting tips that often result from tip preparation on these surfaces. Therefore, in order to check the consistency of our observations, we repeated our measurements for $\mathrm{C}_{60}$ adsorbed on the metallic $\mathrm{Ag}(111)$ substrate. Here, in the absence of the well-separated $\mathrm{Si}(111)-7 \times 7$ adatoms and cornerholes as a ready inverse imaging tool, we used a combination of STM and NC-AFM imaging, in conjunction with site-specific force measurements, to identify the tip termination. We note that when performing simultaneous tunnel current measurements we ensure low biases and currents to avoid issues with electronic crosstalk [37].

In Fig. 5 we present imaging and force spectroscopy performed on $\mathrm{C}_{60}$ islands on $\mathrm{Ag}(111)$. Prior to these datasets the tip was heavily indented into the $\mathrm{Ag}$ surface, and therefore it is highly likely the tip was (bulk) Ag terminated. $F(z)$ measurements performed with metal-terminated tips [Figs. 5(a), 5(b), 5(e), and 5(f)] show forces on the order of 0.6-1 nN [Fig. 5(m)], which is in agreement with previous metal probe-fullerene force measurements [11]. In addition to the large tip-molecule interaction, the interpretation of the tips as metal-terminated is supported by their ability to pick up the $\mathrm{C}_{60}$ molecules from the island after close approach [Figs. 5(c), 5(d), 5(g), and 5(h)]. After the removal of the molecules the characteristics of the tip change, with peak tip-sample forces of order $300 \mathrm{pN}$ [Fig. 5(m)], in line with previous measurements of the $\mathrm{C}_{60}-\mathrm{C}_{60}$ interaction $[10,11,34]-$ strongly suggesting that the tip has become $\mathrm{C}_{60}$ terminated.

We also observed cases where the tip appears to have been spontaneously functionalized with a small passivating group such as CO [9] [Figs. 5(i)-5(1)]. We make this assignment based on the clear "bondlike" imaging in the constant height $\Delta f$ images, the current contrast which bears a striking resemblance to the current images collected with $\mathrm{CO}$ tips and during STHM imaging [38-40], and the small peak tip-sample force $(\sim 100 \mathrm{pN})$ which is in line with previous measurements for $\mathrm{CO}$ tips on $\mathrm{C}_{60}$ and other organic molecules adsorbed on $\mathrm{Cu}(111)[9,28]$. In passing we note that these data suggest that some results previously assigned to metal-terminated tips [41], which demonstrated an abnormally small tip-sample attractive interaction, may in fact have resulted from molecularly terminated tips. Our results on the $\operatorname{Ag}(111)$ surface are in line with previous metal-molecule measurements, and consequently suggest that our silicon-molecule force measurements are correct, and do not result from an error in calibration or misassignment of the tip termination.

\section{DISCUSSION}

The most surprising outcome of these experiments is that many $\mathrm{Si}$ cluster-fullerene interactions are almost identical in magnitude to the fullerene-fullerene and $\mathrm{CO}-\mathrm{C}_{60}$ interaction, despite the silicon material having a nominally higher reactivity. In particular, it is counterintuitive that the silicon cluster-molecule interaction is significantly weaker than the metal cluster-molecule interaction, considering the considerably higher reactivity of silicon surfaces compared to noble metal surfaces. However, it is important to note that one cannot consider the reactivity of any tip (or indeed any substance) in isolation. As is well established in many fields of chemistry, while some substances are generally to be considered more reactive than others, it is always necessary to consider both reactants and, in particular, the precise local atomic structure of the materials, before any statement can be made as to whether a reaction will occur in a given case. Hence, as our simulations, and prior theoretical work investigating atomic scale contrast formation in scanning probe investigations of graphite and carbon nanotubes [12], suggest, although the pristine $\operatorname{Si}(111)-7 \times 7$ surface is highly reactive, disordered silicon structures that form at the apex of a scanning probe can be considerably less so. It appears that only the most reactive, highly oriented silicon clusters are capable of rehybridizing the $\mathrm{C}-\mathrm{C}$ bond, whereas (perhaps more realistic) dimerlike tips do not react strongly with closed carbon structures. Further combined experimental and theoretical support for our interpretation can be found in recent work showing intramolecular resolution on silicon surfaces [2,4], where similar DFT calculations suggested that dimerlike tips should also react relatively weakly with small organic molecules (such as naphthalenetetracarboxylic diimide (NTCDI) and perylenetetracarboxylic dianhydride (PTCDA)) adsorbed on the $\operatorname{Si}(111)-7 \times 7$ surface.

\section{CONCLUSIONS}

In conclusion, we have shown experimentally that, counterintuitively, many silicon-terminated tips interact exceptionally weakly with the $\mathrm{C}_{60}$ molecule, and can enter into the repulsive regime to allow intramolecular imaging. The silicon termination was confirmed by careful experimental design and inverse imaging on silicon adatoms. The interaction is quantitatively similar to the interaction reported for molecule-terminated tips, but radically different for the interaction between the same molecules and highly oriented silicon clusters. These results highlight the important differences between the properties of the "ideal" surfaces of bulk crystals, and those of small disordered clusters of the same material. Our results are cross-checked with measurements on a noble metal surface that are in line with previous results in the literature, and show surprisingly that noble metal tips react more strongly with small organic molecules than silicon tips. These results have important implications for intramolecular imaging, and also the understanding of reactions involving nanoclusters and disordered nanostructured materials, such as those now commonly used in catalysis.

\section{ACKNOWLEDGMENTS}

A.S. gratefully acknowledges the support of the Leverhulme Trust via Fellowship No. ECF-2013-525. P.J.M. thanks the Engineering and Physical Sciences Research Council and the Leverhulme Trust, respectively, for Grants No. EP/G007837/1 and No. F00/114 BI. P.R. received funding from the People Programme (Marie Curie Actions) of the European Union's Seventh Framework Programme (Grant No. FP7/2007-2013) under Research Executive Agency (REA) Grant No. 628439. S.P.J. thanks the Leverhulme Trust for support via Fellowship No. ECF-2015-005. 
[1] Leo Gross, Fabian Mohn, Nikolaj Moll, Peter Liljeroth, and Gerhard Meyer, Science 325, 1110 (2009).

[2] Adam Sweetman, Samuel P. Jarvis, Philipp Rahe, Neil R. Champness, Lev Kantorovich, and Philip Moriarty, Phys. Rev. B 90, 165425 (2014).

[3] César Moreno, Oleksandr Stetsovych, Tomoko K. Shimizu, and Oscar Custance, Nano Lett. 15, 2257 (2015).

[4] Kota Iwata, Shiro Yamazaki, Pingo Mutombo, Prokop Hapala, Martin Ondráček, Pavel Jelínek, and Yoshiaki Sugimoto, Nature Communications 6, 7766 (2015).

[5] R. Pérez, M. C. Payne, I. Štich, and K. Terakura, Phys. Rev. Lett. 78, 678 (1997).

[6] R. Perez, I. Stich, M. C. Payne, and K. Terakura, Phys. Rev. B 58, 10835 (1998).

[7] P. Pou, S. A. Ghasemi, P. Jelinek, T. Lenosky, S. Goedecker, and R. Perez, Nanotechnology 20, 264015 (2009).

[8] M. A. Lantz, H. J. Hug, R. Hoffmann, P. J. A. van Schendel, P. Kappenberger, S. Martin, A. Baratoff, H.-J. Guntherodt, and H. J. Güntherodt, Science 291, 2580 (2001).

[9] L. Gross, F. Mohn, N. Moll, B. Schuler, A. Criado, E. Guitian, D. Pena, A. Gourdon, and G. Meyer, Science 337, 1326 (2012).

[10] C. Chiutu, A. M. Sweetman, A. J. Lakin, A. Stannard, S. Jarvis, L. Kantorovich, J. L. Dunn, and P. Moriarty, Phys. Rev. Lett. 108, 268302 (2012).

[11] Nadine Hauptmann, Fabian Mohn, Leo Gross, Gerhard Meyer, Thomas Frederiksen, and Richard Berndt, New J. Phys. 14, 073032 (2012).

[12] Martin Ondráček, Pablo Pou, Vít Rozsíval, Cesar González, Pavel Jelínek, and Rubén Pérez, Phys. Rev. Lett. 106, 176101 (2011).

[13] Makoto Ashino, Alexander Schwarz, Timo Behnke, and Roland Wiesendanger, Phys. Rev. Lett. 93, 136101 (2004).

[14] Philip J. Moriarty, Surf. Sci. Rep. 65, 175 (2010).

[15] Certain commercial equipment, instruments, or materials (or suppliers, or software, etc.) are identified in this paper to foster understanding. Such identification does not imply recommendation or endorsement by the National Institute of Standards and Technology, nor does it imply that the materials or equipment identified are necessarily the best available for the purpose.

[16] Adam Sweetman and Andrew Stannard, Beilstein Journal of Nanotechnology 5, 386 (2014).

[17] John E. Sader and Suzanne P. Jarvis, Appl. Phys. Lett. 84, 1801 (2004).

[18] J. Hutter, M. Iannuzzi, F. Schiffmann, and J. VandeVondele, WIREs Comput. Mol. Sci. 4, 15 (2014).

[19] J. VandeVondele, M. Krackb, F. Mohamedb, M. Parrinellob, T. Chassaingc, and J. Hutterc, Comput. Phys. Commun. 167, 103 (2005).
[20] G. Lippert, J. Hutter, and M. Parrinello, Mol. Phys. 92, 477 (1997).

[21] M. Hutter J. Goedecker, and S. Teter, Phys. Rev. B 54, 1703 (1996).

[22] K. Ernzerhof, M. Perdew, and J. P. Burke, Phys. Rev. Lett. 77, 3865 (1996).

[23] S. Grimme, J. Antony, S. Ehrlich, and H. Krieg, J. Chem. Phys. 132, 154104 (2010).

[24] J. VandeVondele and J. Hutter, J. Chem. Phys. 127, 114105 (2007).

[25] Raw data may be found at http://dx.doi.org/10.17639/nott.55.

[26] Mark P. Boneschanscher, Joost Van Der Lit, Zhixiang Sun, Ingmar Swart, Peter Liljeroth, and Daniël Vanmaekelbergh, ACS Nano 6, 10216 (2012).

[27] Masayuki Abe, Yoshiaki Sugimoto, O. Custance, S. Morita et al., Appl. Phys. Lett. 87, 173503 (2005).

[28] Leo Gross (private communication, 2015).

[29] L. Gross, N. Moll, F. Mohn, A. Curioni, G. Meyer, F. Hanke, and M. Persson, Phys. Rev. Lett. 107, 086101 (2011).

[30] Andrew J. Lakin, Cristina Chiutu, Adam M. Sweetman, Philip Moriarty, and Janette L. Dunn, Phys. Rev. B 88, 035447 (2013).

[31] Ayhan Yurtsever, Yoshiaki Sugimoto, Hideki Tanaka, Masayuki Abe, Seizo Morita, Martin Ondráček, Pablo Pou, Rubén Pérez, and Pavel Jelínek, Phys. Rev. B 87, 155403 (2013).

[32] Adam Sweetman, Philipp Rahe, and Philip Moriarty, Nano Lett. 14, 2265 (2014).

[33] Peter Sharp, Sam Jarvis, Richard Woolley, Adam Sweetman, Lev Kantorovich, Chris Pakes, and Philip Moriarty, Appl. Phys. Lett. 100, 233120 (2012).

[34] Adam Sweetman, Rashid Mohammad, Samuel Jarvis, Philipp Rahe, Janette Dunn, and Philip Moriarty (unpublished).

[35] Anna Campbellová, Martin Ondráček, Pablo Pou, Rubén Pérez, Petr Klapetek, and Pavel Jelínek, Nanotechnology 22, 295710 (2011).

[36] A. Sweetman, S. Jarvis, R. Danza, J. Bamidele, S. Gangopadhyay, G. A. Shaw, L. Kantorovich, and P. Moriarty, Phys. Rev. Lett. 106, 136101 (2011).

[37] Zsolt Majzik, Martin Setvín, Andreas Bettac, Albrecht Feltz, Vladimír Cháb, and Pavel Jelínek, Beilstein Journal of Nanotechnology 3, 249 (2012).

[38] C. Weiss, C. Wagner, C. Kleimann, M. Rohlfing, F. S. Tautz, and R. Temirov, Phys. Rev. Lett. 105, 086103 (2010).

[39] R. Temirov, S. Soubatch, O. Neucheva, A. C. Lassise, and F. S. Tautz, New J. Phys. 10, 053012 (2008).

[40] Leo Gross, Fabian Mohn, Nikolaj Moll, Gerhard Meyer, Rainer Ebel, Wael M. Abdel-Mageed, and Marcel Jaspars, Nature Chemistry 2, 821 (2010).

[41] Rémy Pawlak, Shigeki Kawai, Sweetlana Fremy, Thilo Glatzel, and Ernst Meyer, ACS Nano 5, 6349 (2011). 